Archives

$10 \mid 1993$

Le Centre de recherches historiques de 1949 à 1975

\title{
Origines d'une nouvelle organisation de la recherche en sciences humaines
}

\section{(2) OpenEdition \\ 12 Journals}

Édition électronique

URL : http://journals.openedition.org/ccrh/2785

DOI : $10.4000 /$ ccrh. 2785

ISSN : $1760-7906$

Éditeur

Centre de recherches historiques - EHESS

Édition imprimée

Date de publication : 15 avril 1993

ISSN : 0990-9141

Référence électronique

"Origines d'une nouvelle organisation de la recherche en sciences humaines », Les Cahiers du Centre de Recherches Historiques [En ligne], 10 | 1993, mis en ligne le 16 mars 2009, consulté le 01 mai 2019. URL : http://journals.openedition.org/ccrh/2785 ; DOI : 10.4000/ccrh.2785

Ce document a été généré automatiquement le 1 mai 2019.

Article L.111-1 du Code de la propriété intellectuelle. 


\section{Origines d'une nouvelle organisation de la recherche en sciences humaines}

1 Partons de la dernière mesure de réorganisation institutionnelle des années 1970 : au premier janvier 1979, le CRH constitue un laboratoire mixte EHESS - CNRS qui rassemble 115 chercheurs et techniciens dont 81 de l'École ${ }^{1}$. Ce laboratoire est doté d'une direction, d'un secrétariat, d'un comité de direction et d'un conseil de laboratoire conforme aux normes du CNRS. Il présente tous les traits d'un grand centre de recherche qui fonde son existence sur un personnel de chercheurs et de collaborateurs techniques permanents et spécialisés dans la recherche, d'une direction contrôlée par des représentants du personnel, qui organise ses activités à partir de contrats de collaboration et d'association pluriannuels avec le CNRS ou d'autres organes de financement de la recherche scientifique. La taille du centre impose une gestion routinière et pratiquement bureaucratique de ses activités scientifiques qui sont d'ailleurs bien intégrées dans le champ de recherche historique universitaire: en 1976, le CRH n'est qu'un des 6 laboratoires associés du CNRS dans le domaine de l'histoire, auxquels il faut ajouter les trois centres propres du CNRS et les 29 équipes de recherche associées qui s'occupent d'archéologie ou d'histoire ${ }^{2}$. Ces unités liées au CNRS figurent aussi parmi les plus grandes des 254 unités de recherche à dominante historique que le fichier du Centre de documentation en sciences humaines de la MSH a inventoriées pour $1980^{3}$. Dans le cas du CRH, son association institutionnelle au CNRS remonte à 1967, année où il se constitue en laboratoire associé $\mathrm{n}^{\circ} 93 \mathrm{du}$ CNRS. Cette association régularise une coopération de fait qui commence avec la création du CRH en 1949 : tout au long de son existence, le CRH a eu recours aux crédits du CNRS et a profité de la collaboration de son personnel.

2 Le fait qu'aujourd'hui les centres de recherches et les laboratoires, sont devenus une forme commune de la recherche en sciences humaines, fait souvent oublier que les débuts de cette forme organisationnelle de la recherche historique ne remontent pas loin dans le passé : la plupart des unités de recherche existant en 1979 furent crées après 1960, un grand nombre après les réformes et restructurations de la fin des années 1960. L'idée de transférer le modèle du laboratoire dans les sciences humaines s'est diffusé dans l'entredeux-guerres. C'est alors qu'on vit naître les premiers centres d'une certaine grandeur qui concentraient leurs efforts sur la recherche et qui dépassaient le cadre d'un séminaire 
et d'une bibliothèque spécialisée à l'intérieur de l'université ou d'un autre établissement d'enseignement supérieur. D'abord favorisée par les disciplines spécialisées de l'histoire qui n'occupaient qu'une place marginale dans le système d'enseignement supérieur (Institut de paléontologie humaine, 1914 ; Institut d'études islamiques de l'université de Paris, 1929 ; Institut d'histoire des sciences et des techniques de l'université de Paris, 1932), la nouvelle forme d'organisation fut adoptée dès les années 1930 au coeur même de la discipline. En 1936, fut créé l'Institut d'histoire des relations internationales contemporaines de l'université de Paris; en 1937 suivaient l'institut d'histoire de la révolution française à la Sorbonne et l'Institut de Recherche et d'Histoire des Textes (IRHT) du CNRS. Ce dernier représentait le plus clairement la nouvelle tendance à confier "la besogne ingrate, mais féconde de l'équipement scientifique en histoire ${ }^{4}$ » à des institutions de recherche dotées d'un personnel spécialisé permanent et d'un équipement matériel approprié. Parmi les historiens, l'expérience de la crise de l'édition scientifique de documents historiques pendant l'entre-deux-guerres avait préparé un milieu plutôt individualiste et hostile à la recherche collective à accepter des nouvelles formes d'organisation. Les difficultés qu'ont rencontré les projets d'éditions de sources des sociétés savantes et du Comité des travaux historiques et scientifiques pendant cette période ont fait voir les limites financières et sociales des formes d'organisation de la recherche établies au dix neuvième siècle, qui reposaient sur la participation d'une couche aisée de notables locaux, sur un système de financement publique lacunaire et sur le travail individuel d'érudits et d'historiens responsables des éditions ${ }^{5}$.

Pendant l'entre-deux-guerres, le modèle traditionnel de l'organisation de la recherche historique devenait la cible de critiques internes que les hérétiques des Annales d'histoire économique et sociale ou de la Revue de synthèse présentaient à leurs collègues.

Lucien Febvre est parti d'une réflexion sur les révolutions dans les sciences naturelles pour plaider en faveur d'un aggiornamento des habitudes de recherche en histoire. Pour lui, la recherche collective n'était pas seulement un moyen de rationaliser les efforts, de continuer les travaux classiques d'érudition avec des moyens plus modernes en réduisant les gaspillages. Dans sa vision utopique d'une historiographie renouvelée, la réorganisation de la recherche était l'occasion de rompre avec les anciennes croyances méthodologiques du métier, à savoir le modèle descriptif et les convictions empiristes. Par contre, la recherche collective offrait le moyen de familiariser le milieu des historiens avec un modèle constructiviste et interdisciplinaire de la recherche. " $\mathrm{Si}$, ayant choisi, pour des raisons valables, un sujet d'études ; l'ayant délimité avec soin ; ayant marqué ce qu'avant tout il importait d'arriver à établir [...] - [l'historien] organisait les recherches d'une équipe composée, disons (en songeant à certaines enquêtes possibles et désirables d'histoire des techniques) d'un technicien proprement dit ; d'un chimiste au courant de l'histoire de sa science; d'un économiste à l'esprit concret - si, se réservant le rôle entre tous difficile de dresser les problématiques préalables; de rapprocher les réponses fournies, d'en dégager les éléments de solution, [...] si, ayant pris cette voie longue, qui finalement se trouverait beaucoup plus courte que les vieux chemins sinueux d'autrefois, il parvenait à faire enfin de l'histoire une «science de problèmes à poser», sinon toujours à résoudre avec certitude et du premier coup : je crois que son rôle serait singulièrement plus en vue que celui d'un vague fabricant de livres personnels $[. . .]^{6}$.» Les enquêtes lancées par les Annales d'histoire économique et sociale sur les plans parcellaires en Europe ${ }^{7}$ ou sur les aristocraties européennes ${ }^{8}$ propageaient l'idée d'une recherche approfondie organisée en équipes. Dans le domaine de l'ethnographie populaire, la Commission des 
Recherches Collectives du Comité de l'Encyclopédie Française a organisé ad hoc un travail collectif sur les forges de villages en France9.

Après sa nomination à la Sorbonne, M. Bloch fonda, en 1938, en collaboration avec M. Halbwachs l'Institut d'histoire économique et sociale ${ }^{10}$ où le travail scientifique ne put commencer véritablement qu'après la guerre sous la direction de son successeur $\mathrm{E}$. Labrousse.

Dans la situation de l'après-guerre, tout le contexte politique et culturel était favorable au discours réformateur en matière d'organisation de la recherche scientifique. Les réticences $\mathrm{du}$ milieu universitaire des facultés de lettres furent largement contrebalancées par le soutien des administrateurs politiques et par le poids institutionnel des formes d'organisation de la recherche dans les sciences naturelles qui dominaient largement le CNRS. La création de la $\mathrm{VI}^{\mathrm{e}}$ Section de l'EPHE et, en 1949, le démarrage du CRH s'inscrivent (avec 5 autres centres de recherche historique) dans la vague des réformes de l'immédiat après-guerre.

7 Dans cette liaison étroite d'une forme organisationnelle à des impératifs de recherche spécifiques on peut identifier un élément de ce que Bourdieu a appelé l'effet d'institution ${ }^{11}$ « en parlant de toute la VI ${ }^{\mathrm{e}}$ Section dans les années 1950 et 1960. L. Febvre ne perdait aucune occasion pour créer un tel « patriotisme d'institution ${ }^{12}$ »:

La rubrique « Recherches collectives » est ouverte aux Annales. Je n'ai pas besoin de rappeler à nos amis que c'est une "rubrique maison » et que, dans une certaine leçon inaugurale de 1934 au collège de France, puis dans l'oeuvre d'une première commission des Recherches collectives orientée dans le sens folklorique et animée par André Varagnac, les réalisations actuelles de notre jeune Sixième Section ont reçu avant la lettre une doctrine et des modèles ${ }^{13}$.

8 Nous allons analyser tout de suite la dynamique scientifique qu'un tel «esprit de l'institution » a engendrée, mais il me semble nécessaire de souligner ici la surcharge symbolique que le CRH en tant qu'institution a hérité de ses origines. L'ambition de Febvre et Braudel - monter un laboratoire d'une nouvelle histoire - le liera pour toute la période considérée à des positions de "pointe», de "nouveauté " en matière de méthodes de recherche, de techniques d'enquêtes et de types de documents à dépouiller. Les présentations officielles ont largement puisé dans le mythe d'avant-garde pour marquer l'identité du Centre, comme en témoignent les citations suivantes.

9 «Créé par MM. Febvre et Braudel dans le prolongement de la VI ${ }^{e}$ Section, le Centre de recherches historiques a connu des débuts modestes, puis une extension croissante qui en fait aujourd'hui, dans le domaine de l'histoire économique et sociale, un organisme original, unique encore en France et fort connu au-delà de nos frontières ${ }^{14} »$ (1959).

Il n'est ni exagéré, ni tautologique d'affirmer que le Centre de recherches historiques de la VIe Section de l'EPHE a été ces dernières années au centre et au coeur de la recherche historique française et internationale ${ }^{15}$ (1965).

Il Centre ha assolto a più riprese la funzione di laboratorio e di campo d'esperienza : [...] ora il Centre, come in certo qual modo anche il Warburg Institute ad esempio, ha voluto imboccare una strada ben diversa: e ci sembra che sia riuscito sufficientemente a dimostrare - non solo con la rapidità esteriore de 1 suo sviluppo - quale diversa capacità di accelerazione acquistava la ricerca una volta impostata su di un piano collettivo o di équipe ${ }^{16}$ (1967).

Le Centre de recherches historiques centralise, coordonne et aide les recherches d'un certain nombre d'historiens qui se réclament de la tradition ouverte par Marc Bloch, Lucien Febvre, Ernest Labrousse, Fernand Braudel et de ce qu'on appelle l'École des Annales. [...] Grâce à la révolution introduite par l'ordinateur dans le 
maniement de l'information, il développe aujourd'hui son activité vers le dépouillement de plus en plus systématique des données quantitatives de l'histoire, et la mise au point des procédures d'exploitation les plus sûres et les plus rapides ${ }^{17}$ (1969).

Tableau 1. Création de Centres de recherche en Histoire

\begin{tabular}{|c|c|c|}
\hline Aruneses & Hombre & Extmples \\
\hline $\begin{array}{l}1918 \\
194 \\
194\end{array}$ & 1. 10 & 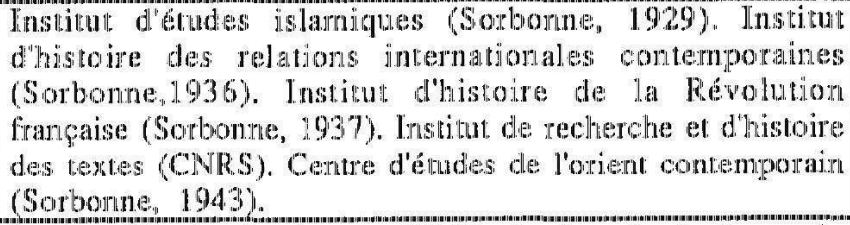 \\
\hline $\begin{array}{l}1945 \\
19.49\end{array}$ & 6 & 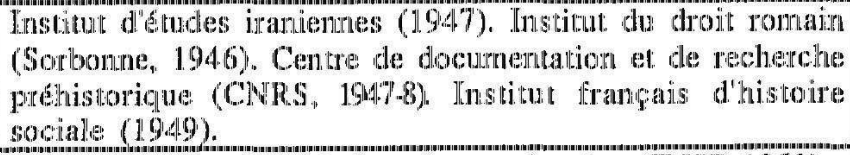 \\
\hline $\begin{array}{l}1951 \\
1956\end{array}$ & 1.5 & 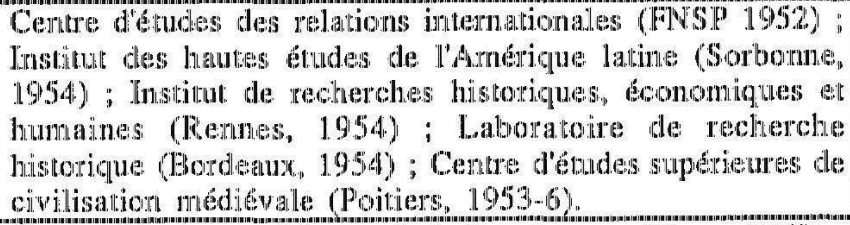 \\
\hline $\begin{array}{l}1957 \\
196 \\
19\end{array}$ & 35 & 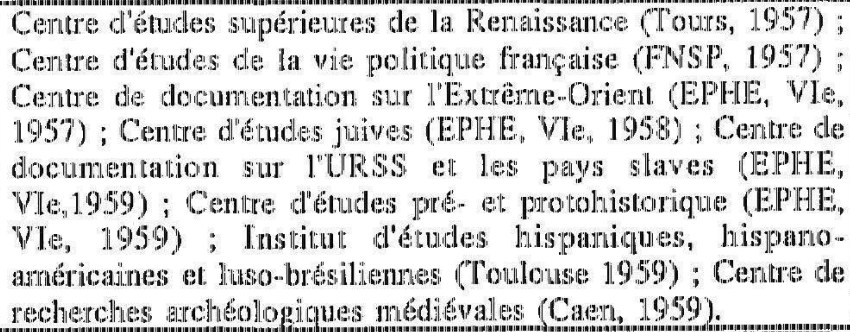 \\
\hline
\end{tabular}

Sources : Répertoire alphabétique des principaux organismes et centres de recherche historique existant en France, in Comité français des sciences historiques, 25 ans de recherche historique en France (1940-1965), p. 67-132. Les exemples mentionnés visent à illustrer les tendances et à indiquer les créations les plus notables.

10 Ces quelques citations illustrent ce type d'auto-présentation qui n'a cessé de mobiliser l'élan du personnel mais qui montrait à la fin des années 1960 des signes de relâchement. Ce qui, au début, était inhérent à la place et à la forme même du Centre - sa position d'avant-garde - devait devenir problématique et finalement un mythe fondateur au fur et à mesure que le modèle organisationnel même se généralisait et que les succès de ses recherches suscitaient des émules plus jeunes et souvent plus dynamiques.

Le tableau 1 fait apparaître une vague de fondations pendant les années 1957-62 qui témoigne $\mathrm{du}$ succès des nouvelles formules organisationnelles, même parmi des historiens toujours hostiles aux innovations programmatiques et sceptiques vis-à-vis d'une recherche collective. Bien que les réalités cachées derrière des noms comme " centre de recherche » ou « institut " puissent être tout à fait différentes, ces trains de création témoignent d'un changement d'orientation dans le monde historiographique universitaire. Le travail en équipe, un programme de recherche bien défini et exigeant un effort prolongé, devenaient des slogans qui faisaient l'unanimité et qui avaient - surtout après 1958 - toutes les chances de trouver des administrateurs politiques favorables et prêts à financer des nouveaux lieux de recherche scientifique ${ }^{18}$. Le bilan des orientations 
de la recherche dressé par J. Schneider, F. Braudel, E. Labrousse et P. Renouvin exprime clairement ce consensus :

La nature et l'objet même des recherches qui se font de nos jours supposent le plus souvent des travaux menés en équipe, dont l'historien le plus individualiste ne peut pas ne pas reconnaître l'urgence et l'utilité. Ces équipes, animées par un maître, ne peuvent travailler qu'à l'intérieur coordonnent les efforts ${ }^{19}$.

À la seule Faculté des Lettres de la Sorbonne, jadis pôle de résistance aux innovations organisationnelles, 7 centres ont été créés entre 1954 et $1960^{20}$. En 1965, l'enquête menée pour le bilan de 25 ans de recherche historique en France fait état de 86 unités de recherche dont la plus grande partie reste de taille modeste (moins de 10 chercheurs ou collaborateurs techniques réguliers hormis les directeurs) d'instituts ou centre. À la seule Faculté des Lettres de la Sorbonne, jadis pôle de résistance aux innovations organisationnelles, 7 centres ont été créés entre 1854 et $1960^{21}$. En 1965, l'enquête menée pour dresser le bilan de 25 ans de recherches historiques en France fait été de 86 unités de recherche dont la plus grande partie reste de taille modeste (moins de 10 chercheurs ou collaborateurs techniques réguliers hormis les directeurs ${ }^{22}$.

L'implantation du nouveau modèle d'organisation de la recherche transformait lentement tout le champ historique universitaire et influait à son tour l'évolution du CRH : La concurrence et la collaboration avec d'autres centres de recherche intervenait de plus en plus aux choix des terrains de recherche et contribuaient ainsi à fixer d'une manière plus ou moins stable la place du Centre à l'intérieur du champ de recherche historique.

13 Le tableau 2 fait voir le réseau de collaboration que le CRH a tissé au cours de son développement. Plus grand et plus ancien que beaucoup des centres provinciaux avec lesquels il collaborait, le CRH apportait souvent son aide financière et profitait à son tour de ses relations pour gérer des enquêtes au niveau national. Dans le cas des centres de Rennes, de Caen ou de Lyon, la collaboration s'est concrétisée en recherches coordonnées ou communes. Cette coopération au niveau d'instituts de recherches était soutenue par un réseau plus fin de coopération continue avec des chercheurs extérieurs. Pendant les années 1950 et 1960, un soutien financier ou une aide matérielle de la part du CRH était souvent à l'origine d'une telle collaboration, à partir de 1965 la coopération de recherches est devenue la règle.

La collaboration et les échanges scientifiques internationaux sont l'autre aspect central des rapports extérieurs du CRH. Le point de départ était le réseau méditerranéen de $\mathrm{F}$. Braudel qui fit venir à Paris des professeurs et chercheurs débutants espagnols, italiens, portugais et yougoslaves tout au long des années 1950 et 1960. Mais c'est sans aucun doute la coopération culturelle franco-polonaise qui a laissé les traces les plus profondes sur le réseau scientifique international du CRH. L'accord avec l'Institut historique de l'Académie polonaise des Sciences de Varsovie institutionnalisa un échange régulier au niveau des chercheurs et prévoyait la venue régulière de boursiers polonais à l'École. La fin des années 1950 et le début des années 1960 furent le temps fort de cet échange. 
Tableau 2. Collaboration du CRH avec d'autres Centres de recherche (avant 1968)

\begin{tabular}{|c|c|}
\hline Nom & Activites \\
\hline $\begin{array}{l}\text { Institut de recherches historiques, } \\
\text { Exconomigues et humaines (Rennes) }\end{array}$ & Enquêtes: Maladies et épidémies.1966 (Meyer) \\
\hline $\begin{array}{l}\text { Centre d'histoire économique et } \\
\text { sociale (Lyon) }\end{array}$ & $\begin{array}{l}\text { Financernent : Enquête sur les sources d'histoire } \\
\text { économique duXVTe siècle.1962-63? (Gascon). }\end{array}$ \\
\hline $\begin{array}{l}\text { Centre d'archeologie médiévale } \\
\text { (Caen) }\end{array}$ & Enquête : Villages disparus. \\
\hline Centre d'histoire quantitative & Enquête : Le bâtiment. \\
\hline $\begin{array}{l}\text { Centre d'éude des sociétés médi- } \\
\text { terranéentes (Aix) }\end{array}$ & $\begin{array}{l}\text { Enquête : Villages disparus. Financenent : } \\
\text { groupe de recherche de Duby } 1959-62\end{array}$ \\
\hline Université de Prifaceton & $\begin{array}{l}\text { Echanges de professeurs dans le cadre des ac- } \\
\text { cords de la VIe Section avec l'Université de } \\
\text { Princeton. }\end{array}$ \\
\hline $\begin{array}{l}\text { Université du Michigan à Ann } \\
\text { Arbor }\end{array}$ & $\begin{array}{l}\text { Projet : Banque de données (Statistique } \\
\text { générale de la France). } 1968 \text { sy. }\end{array}$ \\
\hline $\begin{array}{l}\text { Institut d'Histoire de l'Académie } \\
\text { des Sciences de Pologne }\end{array}$ & $\begin{array}{l}\text { Accord } 1956 \text { : échanges deprofesseurs, bourses } \\
\text { pour des étudiants polonais ;colloques : partici- } \\
\text { pation des archéologues polonais aux fouilles } \\
\text { de l'enquête sur les villages désertés. }\end{array}$ \\
\hline
\end{tabular}

En 1957-58 étaient organisés deux colloques franco-polonais sur l'histoire et ses interprétations (à Cerisy, avec la participation de Gieysztor, Schaff, Kolakowski, Kula, Goldmann, H. Lefevre, R. Aron, H.I. Marrou, J. Romein) et sur les origines des villes polonaises (avec la participation de Francastel, Lombard, Doehard, Ennen, Wolff, Dziewonski, Labuda, Gieysztor, Dembinska, Jadzewski) ${ }^{23}$. L'aide polonaise était décisive pour le démarrage du projet d'archéologie médiévale de l'enquête sur les villages désertés en 1962 destiné à rattraper le retard de l'archéologie française. Les contacts avec les centres de recherche soviétiques, roumains et yougoslaves n'ont jamais atteint l'intensité de cette collaboration. À la fin de l'époque considérée, l'accord de 1975 avec l'Institut d'histoire de l'Académie des Sciences à Budapest institutionnalisa une pratique de coopération avec des chercheurs hongrois qui remontait aux années $1960^{24}$.

16 Entre 1957 et 1965, les contacts du CRH avec les historiens de l'Amérique Latine s'amplifièrent visiblement : l'origine de cet échange, d'ailleurs largement préparé par une politique française d'échanges culturels remontant à l'entre-deux-guerres, était la «découverte ${ }^{25}$ » du cadre américain par l'équipe «Méditerranée » réunie autour de F. Braudel. Des historiens du CRH partirent pour des conférences et des séjours de recherche au Chili, en Argentine, en Uruguay, au Brésil, entraînant à leur tour des historiens et des étudiants d'Amérique Latine: G. Beyhaut enseigna comme directeur associé entre 1964 et 1968 à la division d'histoire de la VI Section.

17 Tout au long des années 1960, le succès international de "l'école historique française » et la croissance du personnel de recherche a élargi le réseau d'échanges scientifiques du $\mathrm{CRH}$ et multiplié les contacts internationaux. On constate que le réseau commence lentement à se déplacer. Le monde anglo-saxon et surtout les États Unis devinrent plus souvent les lieux d'invitation des membres du CRH. Les accords de la $\mathrm{VI}^{\mathrm{e}}$ Section avec l'université de Princeton ouvraient la voie à un échange régulier au niveau des professeurs ; les contacts réguliers avec le Cambridge Group for the History of Population and Social Structure à partir de 1966-67, la coopération avec l'université du Michigan à Ann Arbor pour l'archivage sur ordinateur de la statistique générale de France et avec l'université $\mathrm{du}$ Wisconsin à Madison pour le dépouillement du catasto florentin 
témoignent de cette réorientation des contacts internationaux, une réorientation lente qui ne détruisait pas les autres réseaux noués plus tôt. Au milieu des années 1970, le CRH pouvait profiter à la fois de la politique d'échanges internationaux et de l'ouverture vers l'étranger que la direction de la $\mathrm{VI}^{\mathrm{e}}$ Section a poursuivie dès l'origine de l'institution et de la renommée internationale que « l'école des Annales » venait d'acquérir.

\section{NOTES}

1. CRH, Rapport d'activités 1978-1979-1980. Dactylographié. Paris s.d.

2. CNRS, Laboratoires et services scientifiques. Paris 1976

3. Cité par Michel Vovelle, Rapport sur les disciplines historiques, M. Godelier, les Sciences de l'homme et de la société en France, Paris, 1982, p. 279

4. Ainsi la formule la présentation du Centre de recherche "Lenain de Tillemont» pour l'histoire ancienne du christianisme (La Recherche historique en France 1940-1965, édité par le Comité Français des sciences historiques. Paris, 1965, p. 100).

5. Dumoulin, Profession historien, op. cit. p. 191 et sv.

6. L. Febvre, «Les recherches collectives et l'avenir de l'histoire» (1936), Combats pour l'histoire, Paris, 1956. p. 55-60, à la page 60.

7. Annales d'histoire économique et sociale I, 1929, p. 61 et sv., 225 et sv., 391 et sv., 562 et sv. ; II, 1930, p. 230 et sv,.557 et sv. ; IV, 1932, p. 368 et sv.; V, 1933, p. 150, 152, 374 et sv. ; VI, 1934, p. 51-61.

8. Annales d'histoire économique et sociale, VIII (1936), p. 238-255,.355-378, X(1938), p. 36.

9. André Varagnac, «Une coopérative de travail scientifique : la Commission des Recherches Collectives du Comité de l'Encyclopédie Française ", Annales d'histoire économique et sociale, VII, 1935, p. 302-306

10. C. Fink, Marc Bloch, a life in History, Cambridge 1989. p. 190

11. P. Bourdieu, Homo academicus, op. cit., p. 145.

12. P. Bourdieu, ibid., p. 101.

13. L. Febvre, Annales ESC, 1951, p. 83.

14. VIe Section de l'EPHE, Budget primitif des recettes et des dépenses pour l'exercice 1959, archives EHESS, AJ 66 B 23.

15. CRH, Demande d'association au CNRS, archives EHESS, fonds Velay

16. A. Tenenti, "Quindici anni di attività del Centre de recherches historiques di Parigi ", Studi storici, 1967, p. 203-211, aux pages 204 et 211.

17. CRH, Rapport d'activité 1968-1969, archives EHESS, fonds Velay.

18. D. Ozanam, «Enseignement et recherche en France de l'Ancien Régime à nos jours ", Vingtcinq ans de recherche historique en France (1940-1965), bilan édité par le Comité Français des Sciences historiques, Paris, 1965, p. 3-65, aux pages p. 48 et sv.

19. J. Schneider, F. Braudel, E. Labrousse, P. Renouvin, «Les orientations de la recherche historique ", Revue historique, 1959, T. 222, p. 46

20. En 1954, l'Institut des Hautes Études de l'Amérique Latine ; en 1955, le centre de recherche d'histoire de l'antiquité ; en 1956 le Centre de Recherches d'histoire de l'Art du Moyen âge ; en 1958, le Centre de Recherches d'histoire du Moyen Age et le Centre de Recherches sur la Civilisation de l'Europe Moderne; en 1959, le Centre de Recherche d'histoire de l'Architecture 
moderne; en 1960, le Centre de Recherches d'histoire des Slaves. Sources: CNRS, Rapport national de conjoncture scientifique 1963-64, p. 132, 154 ; Annales de l'université de Paris, 1966, p. 149 et sv.; Comité Français des sciences historiques, La recherche historique en France, op. cit., p. 66-132.

21. En 1954, l'Institut des hautes études de l'Amérique latine; en 1955, le Centre de recherche d'histoire de l'antiquité ; en 1956, le Centre de recherches d'histoire de l'art du Moyen-Âge ; en 1958, le Centre de recherches d'histoire du Moyen-Âge et le Centre de recherches sur la civilisation de l'Europe moderne; en 1959, le Centre de recherches d'histoire de l'architecture moderne ; en 1960, le Centre de recherches d'histoire des Slaves. Sources : CNRS, Rapport national de conjoncture scientifique, 1963/64, p. 132, 154 ; Annales de l'Université de Paris, 1966, p. 149 sv., Comité français des sciences historiques, La recherche historique en France, op. cit., p. 66-132.

22. ibid.

23. VI Section de l'EPHE, budget 1958 (archives EHESS, AJ 66 B 23).

24. Voir, par exemple, le colloque à Budapest en 1968 sur la croissance économique et l'histoire (auquel ont participé Braudel, Furet, Le Roy Ladurie, Duby, Bouvier et Richet du côté français, Pach, Makkai, Fügedi, Kirilly, Kiss, Zumanyi, Velmann et Ranki du côté hongrois).

25. Le terme est de R. Romano, «Encore des illusions », Cahiers Vilfredo Pareto, XXI (1983), p. 201 